\title{
Design and characteristics of hydroxyapatites: effect of radiation
}

Jayati Bhavsar, Areeba Tufail, Pooja Gautam, Brad Arnold, Fow-Sen Choa, et al.

Jayati Bhavsar, Areeba Tufail, Pooja Gautam, Brad Arnold, Fow-Sen Choa, Lisa Kelly, Brian Cullum, Paul Smith, Ching Hua Su, K. D. Mandal, N. B. Singh, "Design and characteristics of hydroxyapatites: effect of radiation," Proc. SPIE 10662, Smart Biomedical and Physiological Sensor Technology XV, 1066205 (14 May 2018); doi: 10.1117/12.2301032

SPIE Event: SPIE Commercial + Scientific Sensing and Imaging, 2018, Orlando, Florida, United States 


\title{
Design and characteristics of hydroxyapatites: Effect of radiation
}

\author{
Jayati Bhavsar, Areeba Tufail, Pooja Gautam*, Brad Arnold, Fow-Sen Choa, Lisa Kelly, Brian \\ Cullum, Paul Smith, Ching Hua Su${ }^{+}$, K. D. Mandal, and N. B. Singh \\ University of Maryland, Baltimore County, Baltimore MD 21250 \\ ${ }^{+}$EM31, NASA Marshall Space Flight Center, Huntsville, AL 35812 \\ "IIT-Banaras Hindu University, Varanasi (UP) India
}

\begin{abstract}
We have prepared silicate based hard materials and have processed it with organic flux. Because of the bioactivities of hydroxyapatites with tissues, this class of materials have attracted interest for bone applications. We have utilized low temperature processing techniques. Organic melt was used and the directional solidification method to cast the shaped sample. This organic treated material has different characteristics than coarsened oxide materials. Our approach involved low temperature processing using nano and micron sized powders of the material system $\mathrm{Na}_{2} \mathrm{O}$ $\mathrm{K}_{2} \mathrm{O}-\mathrm{CaO}-\mathrm{MgO}-\mathrm{Ga}_{2} \mathrm{O}_{3}-\mathrm{SiO}_{2}$, and titanates were processed by sintering and grain growth. Our results indicate that substitution of gallium and magnesium or titanium with some variation in processing methods have great potential to improve the glassy characteristics without decreasing the mechanical properties of bones. Effect of radiation on bone was studied by exposing with commercially available $\mathrm{Cs}^{137}$ gamma ray source. It was observed that electrical resistivity increased due to radiation exposure for this system.
\end{abstract}

Email: $\underline{\text { singna@umbc.edu }}$

Keywords: bones, hydroxyapatite, organic, solidification, oxides

\section{INTRODUCTION}

There is a continuous effort to develop process for tissue engineering for achieving bone compositions both crystalline composites and glasses for good mechanical properties, bioactivity, less degradability, and regeneration characteristics. This can be achieved by both understanding the stoichiometric composition and developing novel processes. Considerable efforts on biodegradable composites have been achieved by synthesizing organic-inorganic composites. The basic idea is to take the advantage of achieving good bone regeneration characteristics in organicinorganic composites by changing their stoichiometric compositions. The apatite type materials (hexagonal, space group $\mathrm{P}_{3 / 6}$ ) are found in nature and have been explored since past half century for their compositions and crystal structures. Hydroxyapatites are excellent materials and play very important role. Previous researches were focused ion calcium based phosphates and some silicates. But there is a great need for substitution of elements such as gallium to replace calcium and applications of silicates and titanates to replace phosphate to achieve slow degradation and to achieve high bone strength. The control of morphology more specifically micro, nanopororous strictures, cellular structures are governed by processes and by choosing proper components. Generally, organics (polymers) have high heat of fusion and grow with facets. It makes control of morphology very difficult. Growth by casting, solution crystallization and other methods make it very difficult. Hydroxyapatites single crystals have been investigated their applications as the laser host material. Czochralksi and flux growth methods have been utilized to achieve single crystals. With the increasing thrust to design hard and active bioactive glasses (BGs), it is important to understand the mechanism of bonding between BGs and soft and hard tissues. Chemical process which involves dissolution and precipitation reactions at BG surfaces upon contact with physiological fluids is very important and most important for regenerative tissues [1-4]. Other factors such as variation[5.6] in $\mathrm{pH}$ due to increased concentration of various ions at the BG-fluid interface affects the morphology at the interface. In many cases microcrystals are developed and provide interface between tissue and new implanted bones. This indicates that variations in ions affect the interactions of BGs with tissue is extremely important factor. Additionally, interaction of rough surfaces of BGs helps in attachment of tissues. The hardening behavior and mechanism and kinetic rate requires more study. The composition and local $\mathrm{pH}$ (and hence ion generation) locally vary a lot. This makes any quantitative prediction on growth rate and kinetics of growth very difficult. 
The objectives of this study were to develop novel composition containing gallium materials and surfaces to prevent wear and corrosion; using nano, micro and bulk materials and novel processes for designing structures to increase fatigue and fracture resistance; understanding the morphology and evaluate thermal properties which may throw light on performance function in that occur because of aging or disease. There are several other outstanding issues such as (a) activity of tissues for bone replacements (b) suitability of crystalline composites vs glass and (c) monolithic vs composites which need to be explored in detail. The focused objectives were to develop soft and hard tissues by doping heavy element (gallium) which may have less depletion compared to calcium as results of aging, understand glassy behavior and effect of radiation on properties.

\section{EXPERIMENTAL METHODS AND RESULTS}

As described in the previous section the goal was to understand the morphology specially to process samples free from precipitates for good, glassy behavior, several compositions were synthesized. The results of two compositions listed in Table 1 are presented in this paper. The focus was to determine the morphology of two gallium containing samples with and without titanium.

Table 1- Composition of two mixtures for synthesis

\begin{tabular}{|l|l|l|}
\hline Material & Sample A Weight (g) & Sample B Weight (g) \\
\hline Sodium carbonate & 0.60 & 0.6 \\
\hline Calcium oxide & 2.0 & 2.0 \\
\hline Magnesium oxide & 0.2 & 0.2 \\
\hline Potassium carbonate & 1.2 & 1.2 \\
\hline Silicon oxide & 5.3 & 5.3 \\
\hline Gallium oxide & 0.4 & 0.4 \\
\hline Titanium oxide & 0.00 & 0.6 \\
\hline
\end{tabular}

2.1 Synthesis of samples: As supplied chemicals (Fig.1a) by Sigma Aldrich were listed for the range of 99+\% purity. No further purification was performed. The stochiometric ration as listed in Table 1 was used to prepare two samples. Samples were mixed and grinded to achieve homogeneous mixture.

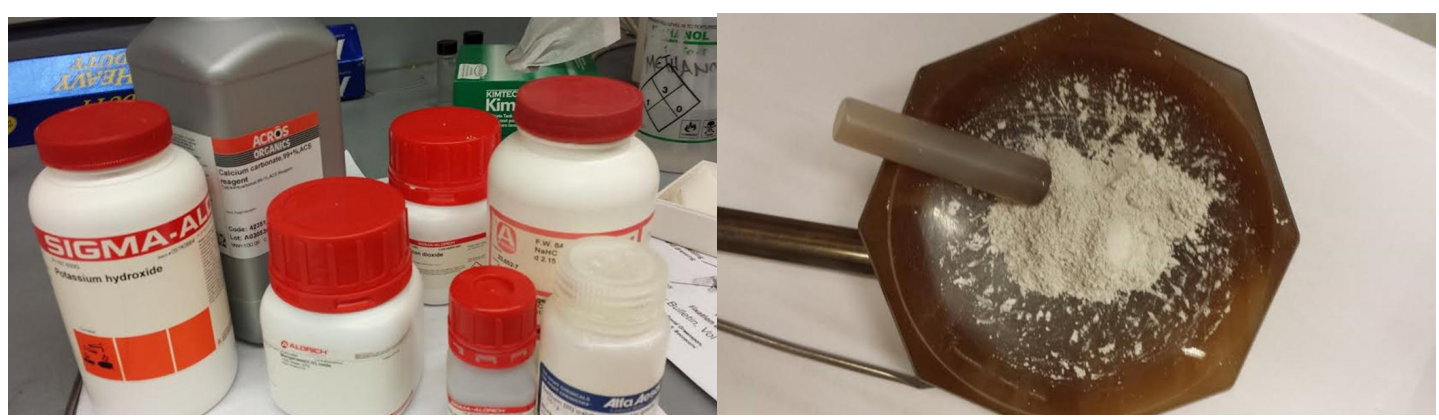

(a)

(b)

Fig. 1 Two compositions of mixtures (a) As supplied chemicals and (b) fine powder of mixture of chemicals.

There were two approaches which we used for the processing of samples. These processes are:

(a) Sintering at high temperature: In the first approach we prepared pressed pellets of samples and annealed these samples at $700 \mathrm{C}$ for a period of 24 hours. The samples were cooled by switching the power of the furnace. There was no control on cooling rate. Since cooling rate has pronounced effect on the morphology and glass formation, this requires further research to understand glassy and crystalline transition behavior in this material system.

(b) Solution process: In this process mixture was placed in a solution of dilute nitric acid-water mixture at room temperature. After an hour of leaving the sample at room temperature, we heated the material and kept in the 
range of $70-80{ }^{\circ} \mathrm{C}$ for several hours. The material does not dissolve completely. This material was heated to driveaway the solvent. Almost after 24 hours the sample dried and was filtered. This sample was also pressed into a pellet and was annealed for 24 hours in a furnace at $700 \mathrm{C}$.

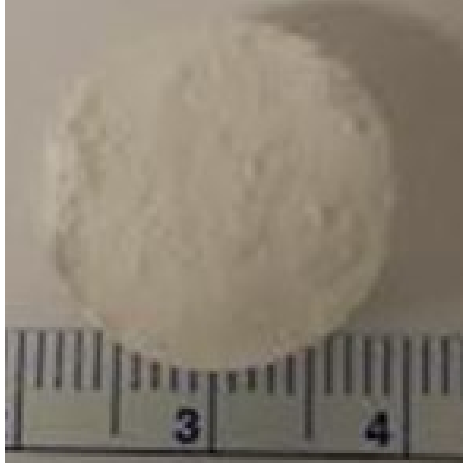

(a)

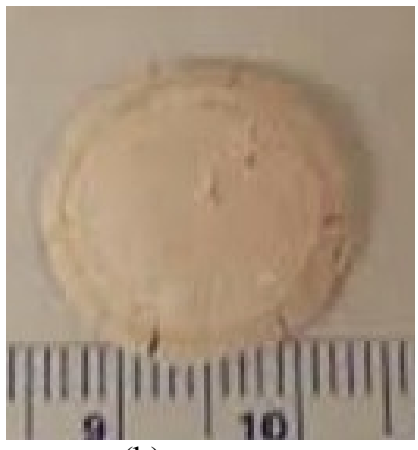

(b)

Fig. 2 Pellets pressed at $8000 \mathrm{psi}$ to prepare $12 \mathrm{~mm}$ diameter samples (a) as prepared and (b) annealed at $700 \mathrm{C}$ pellets

2.2 Morphological Study: The morphology of the material was studied at low magnifications (50-200X) using microscope and scanning electron microscope using a NOVA NANOSEM 450 system for transitioning of particles into glassy morphology. We observed that material processed in solution followed by annealing showed excellent glassy matrix. Fig.3 shows morphology of sample which was placed at $150{ }^{\circ} \mathrm{C}$ for 24 hours. This sample showed some small size precipitates which may have resulted from insoluble species.

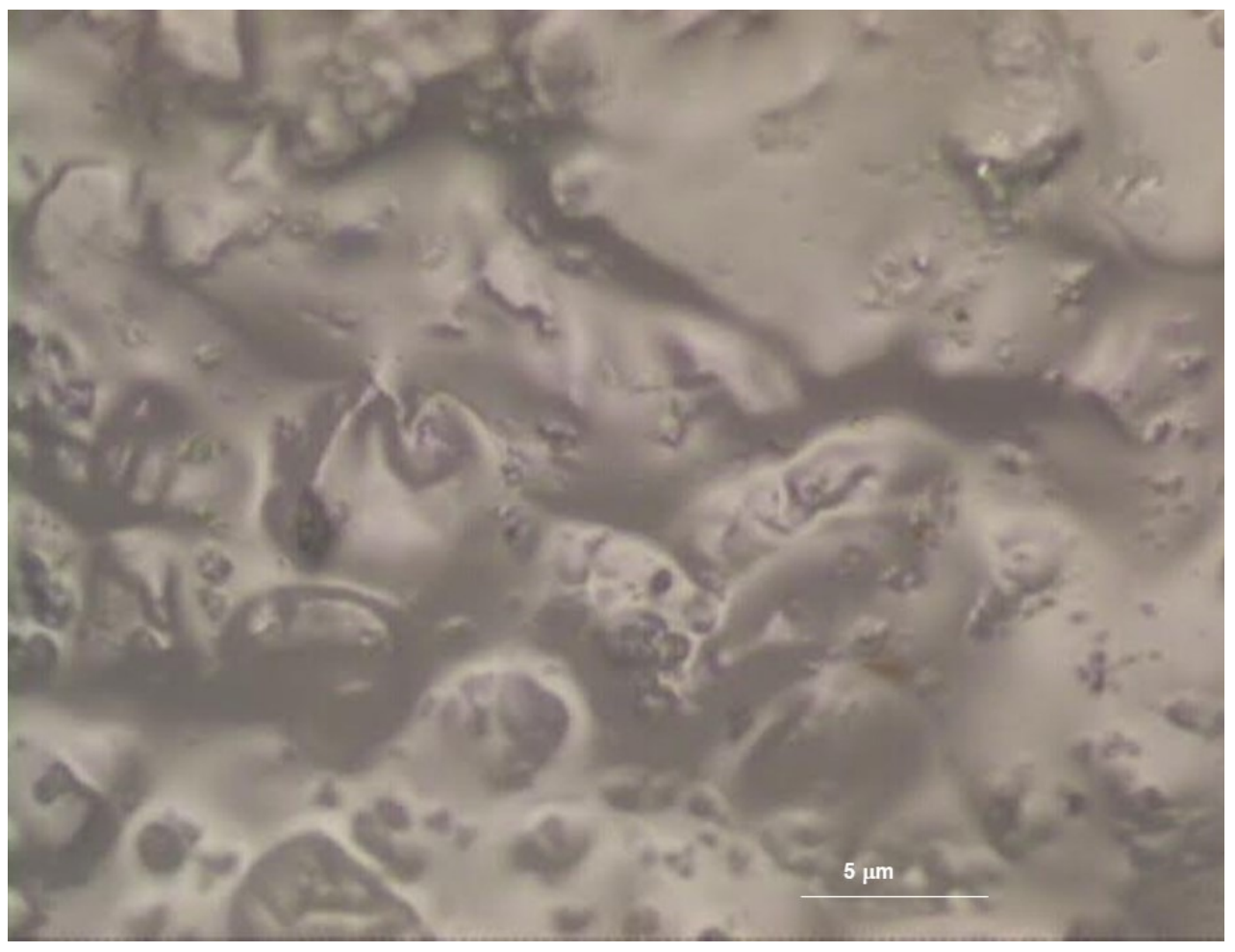

Fig. 3 Sample prepared by solution method followed by drying at 150C showed small precipitates

The sample processed in solution was also used to determine morphological change at $700^{\circ} \mathrm{C}$. Annealing at high temperature dissolved the precipitates. As shown in Fig. 4 there was no sign of precipitates in the matrix. Also, there 
were no faceted grains or large voids and holes in the sample. There was no sign of crystals in this matrix. Also, the surface morphology consisted of smooth layers.

For a comparison both samples were processed for grain growth using ceramic processes. There was no solvent used in this process. Samples were placed at $700^{\circ} \mathrm{C}$ for a period of 50 hours. A typical morphology of sample is shown in Fig. 5. Annealing of $12 \mathrm{~mm}$ diameter and $5 \mathrm{~mm}$ thick transformed completely into hard matrix. Surfaces of this sample were very rough with some hills and valley indicating significant shrinking during the annealing.

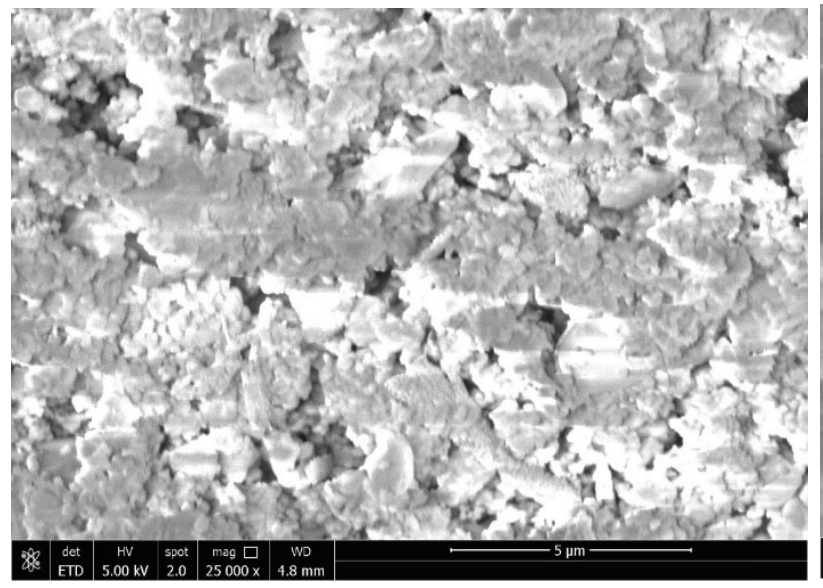

(a)

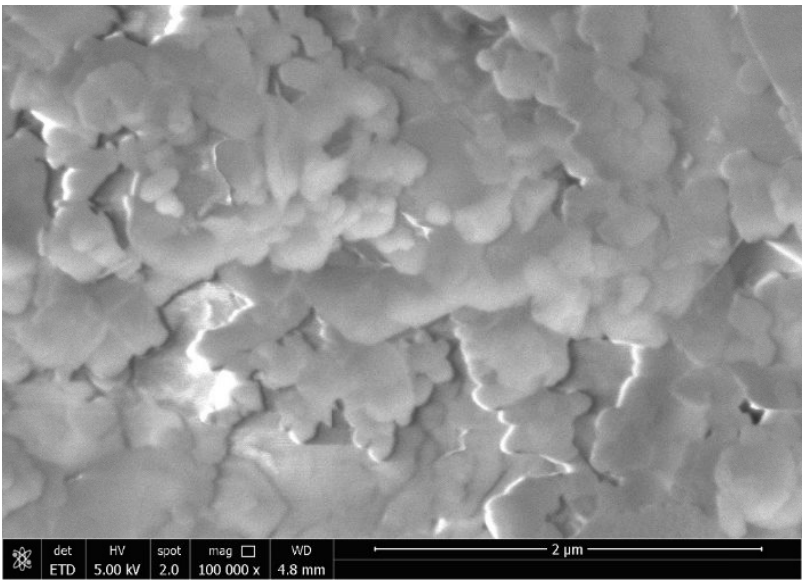

(b)

Fig. 4 Morphology of sample containing titanium showing glassy behavior.

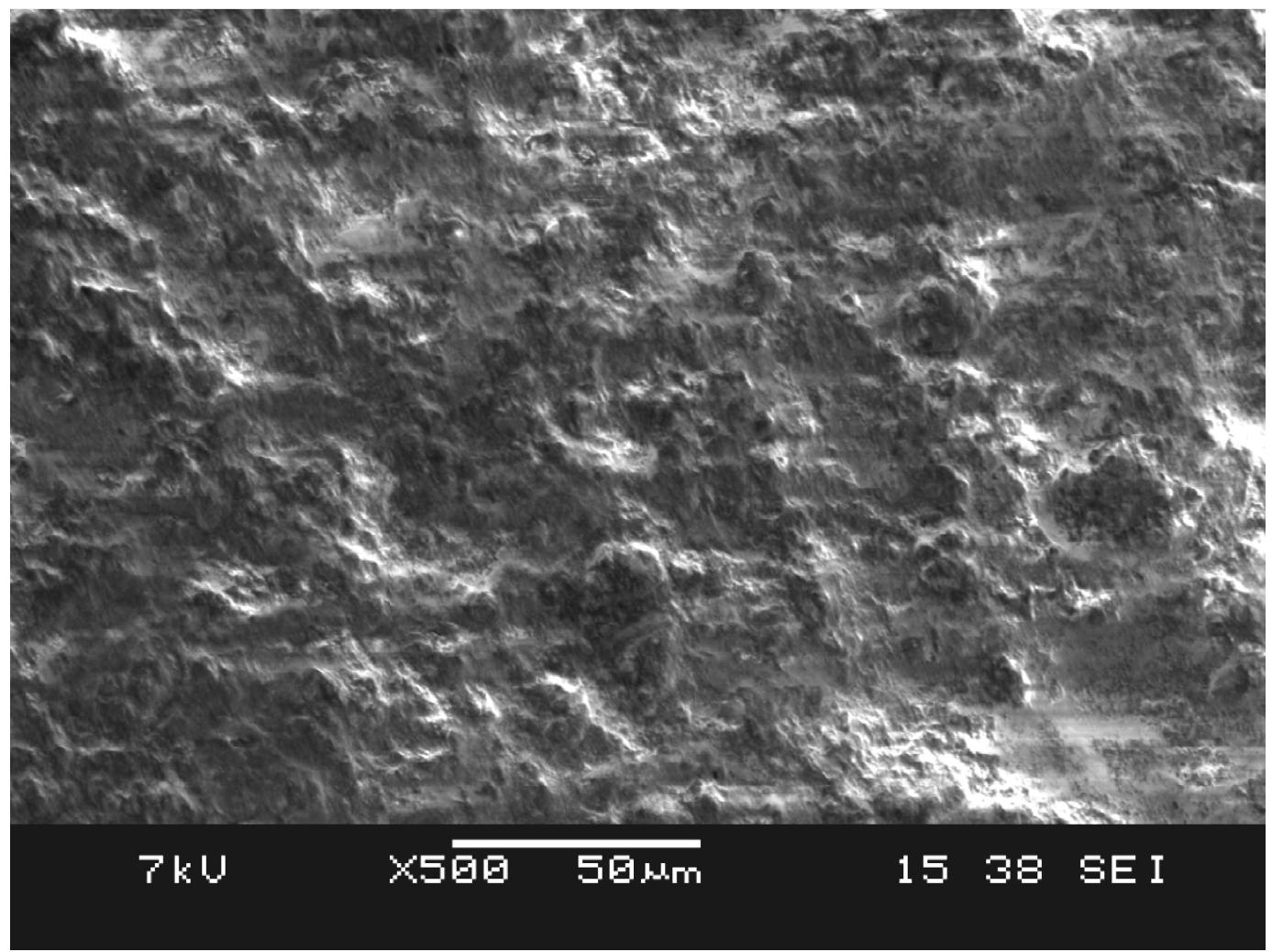

Fig. 5 Sample annealed at $700^{\circ} \mathrm{C}$ did not show voids or any micron size precipitates 
2.4 Bioactivity and effect of radiation: In the field of bio glasses continuous attempts have been made [1-6] to increase the bioactivity by using copper, strontium and other dopants in phosphate composites. We have performed some experiments using both compositions (with and without titanium) to test the validity of organic-inorganic composites for increasing activity. In the preliminary tests we used low temperature organic eutectic mixtures. A as casted sample in eutectic is shown in Fig. 6. This sample has annealed mixture and could be fabricated for desired shapes.

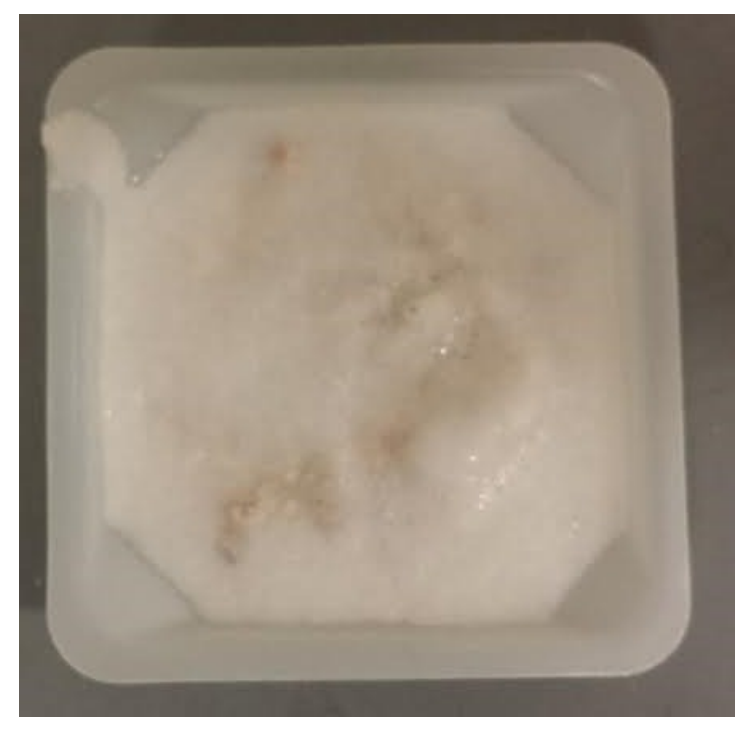

Fig. 9 As casted large $(4 \times 4 \mathrm{~cm})$ sample using low melting organic eutectic. This sample has annealed mixture

For the effect of radiation, we used a commercially available gamma-ray source $\mathrm{Cs}^{137}$. The effect of radiation was estimated by measuring the resistivity of material. The measured resistivity [7] is in the range of $10^{-3} \mathrm{ohm}-\mathrm{cm}$. It was changing significantly in range of $100 \mathrm{~Hz}$ to $1 \mathrm{MHz}$ range. After a radiation of $>50$ hours the resistivity increased significantly for all frequency. This indicates that radiation affects the ionization process of the prepared samples. Further tests are continuing using spectral techniques such as IR and Raman for as prepared and irradiated samples. Also, differential thermal analysis and mechanical tests are continuing.

\section{ACKNOWLEDGEMENT}

The authors would like to acknowledge the support of Space Life and Physical Sciences Division, Human Exploration and Operations Mission Directorate, NASA Headquarter and Marshall Space Flight Center Huntsville Alabama.

\section{SUMMARY}

Two silicate hydroxyapatites were synthesized using solution and annealing methods. It was observed that reactive solution process followed by annealing produced glasses free from precipitates and voids. Effect of radiation on bone was studied by exposing with commercially available $\mathrm{Cs}^{137}$ gamma ray source. It was observed that electrical conductivity decreases due to its exposure. 


\section{REFERENCES}

[1]. L.L Hench, J. Materials Science, mater, Med, 17, 967 (2006).

[2]. Valentina Miguez-Pacheco, David Greenspan, Larry Hench and Aldo R. Boccaccini, American Ceramic Society Bulletin, 94, 27-31 (2015).

[3]. A. Hoppe, N. S. Guldal and A. R. Boccaccini, Biomaterials 32 (11), 2757 (2011).

[4] M. N. Rahaman, D. E. Day, B. S. Bal, Q. Fu, S. B. Jung, L. F. Bonewald and A. P. Tomsia, Acta Biomater., 72355 (2011).

[5]. A. A. Gorustovich, J. A. Roether and A. R. Boccaccini, Tissue Eng, Part B Rev. 16, 2, (2010)199.

[6] Guilin, Luo, Yufei Ma, Xu Cui, Lixin Jiang, Mingming Wu, Yang Hu, Yanfeng Luo, Haobo Pan and Changshun Ruan, RCA Advances, 7, 11880 (2017).

[7]. Areeba Tufail, Christopher Cooper, Stacey Sova, Eric Bowman, Bradley Arnold, Fow-Sen Choa, Brian Cullum, K. D. Mandal and N. B. Singh, Effect of radiation on bone materials" (To be communicated) 